\title{
RELATOS DE PESQUISAS \\ CARACTERIZAÇÃO DO PROCESSO DE GESTÃO DE IDEIAS NO CONTEXTO DO FRONT END DA INOVAÇÃO: UMA REVISÃO INTEGRATIVA
}

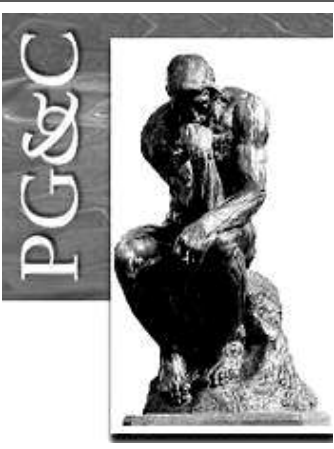

\author{
Aline de Brittos Valdati \\ Doutoranda em Engenharia e Gestão do conhecimento pela Universidade \\ Federal de Santa Catarina, Brasil. \\ E-mail: alinevaldati@gmail.com
}

João Artur de Souza

Doutor em Engenharia de Produção pela Universidade Federal de Santa Catarina, Brasil. Professor da Universidade Federal de Santa Catarina, Brasil.

E-mail: jartur@gmail.com

\author{
André de Oliveira Leite \\ Mestre em Engenharia e Gestão do Conhecimento pela Universidade \\ Federal de Santa Catarina, Brasil. \\ E-mal: andreredes@gmail.com \\ Gregório Jean Varvakis Rados \\ Doutor em Manufacturing Engineering pela Loughborough University of Technology, \\ Inglaterra. Professor da Universidade Federal de Santa Catarina, Brasil. \\ E-mail: grego@egc.ufsc.br
}

\begin{abstract}
Resumo
A gestão de ideias consolida-se na literatura, como um dos fatores para obter-se sucesso em projetos de inovação. Novos produtos normalmente advêm de uma ideia, por isso as organizações buscam formas de organizar essas ideias a fim de alimentar o processo de inovação. No entanto, essa não é uma tarefa fácil devido ao ambiente a qual está inserida. $O$ ambiente nas fases iniciais de inovação é caracterizado por incertezas e exige um equilíbrio entre formalização e criação livre. A pesquisa objetiva identificar os elementos que caracterizam o processo de gestão de ideias com foco no front end da inovação. Para isso realiza-se uma revisão integrativa da literatura sobre gestão de ideias utilizando as principais bases de dados internacionais multidisciplinares, seguindo um protocolo de sete etapas. Como resultado a pesquisa encontra seis fases principais para a gestão de ideias, são elas: 1) preparação, 2) geração e coleta, 3) filtragem, agrupamento e categorização, 4) enriquecimento, 5) avaliação, seleção e priorização e 6) refinamento. Para cada fase aponta os atores envolvidos, bem como o resultado pretendido. Além disso, outros elementos identificados e considerados essenciais foram o "armazenamento" e o "feedback e acompanhamento". Por fim, a pesquisa conclui como oportunidades de estudos futuros, uma matriz de função para a gestão de ideias, o papel da gestão do conhecimento como apoiadora e a pesquisa de fases menos exploradas na literatura. Como a preparação da organização para receber e implementar iniciativas de gestão de ideias e como realizar a avaliação e acompanhamento dessas iniciativas.
\end{abstract}

Palavras-chave: Inovação. Gestão de Ideias. Front end da inovação.

Perspectivas em Gestão \& Conhecimento, João Pessoa, v. 10, n. 3, p. 205-225, set./dez. 2020. DOI: https://dx.doi.org/10.22478/ufpb.2236-417X.2020v10n3.46889

http://periodicos.ufpb.br/ojs2/index.php/pgc. ISSN: 2236-417X. Publicação sob Licença (cc) EY-Nc-ND 


\title{
CHARACTERIZATION OF THE IDEAS MANAGEMENT PROCESS IN THE FRONT END CONTEXT OF INNOVATION: AN INTEGRATING REVIEW
}

\begin{abstract}
The idea management is consolidated in the literature, as one of the factors to obtain success in innovation projects. New products usually come from an idea, so organizations look for ways to organize those ideas in order to feed the innovation process. However, this is not an easy task due to the environment in which it is inserted. The environment in the early stages of innovation is characterized by uncertainties and requires a balance between formalization and free creation. The research aims to identify the elements that characterize the idea management process with a focus on the innovation front end. For this, an integrative review of the literature on the management of ideas is carried out using the main international multidisciplinary databases, following a seven-step protocol. As a result, the research finds six main phases for the management of ideas, they are: 1) preparation, 2) generation and collection, 3) filtering, grouping and categorization, 4) enrichment, 5) evaluation, selection and prioritization and 6) refinement. For each phase it points out the actors involved, as well as the intended result. In addition, other elements identified and considered essential were "storage" and "feedback and monitoring". Finally, the research concludes as opportunities for future studies, a function matrix for the idea management, the role of knowledge management as a supporter and the research of less explored phases in the literature. How to prepare the organization to receive and implement idea management initiatives and how to carry out the evaluation and monitoring of these initiatives.
\end{abstract}

Keywords: Innovation. Idea Management. Front End Innovation.

\section{INTRODUÇÃO}

A competitividade cada vez maior, faz com que os ciclos de novos produtos encurtem, o que aumenta a necessidade de gerar inovações cada vez mais rápidas (HORTON, GOERS, 2014). Nesse sentido, corroborando com os autores Brennan e Dooley (2005) e Aggard (2013), o estímulo a inovação depende da capacidade que a organização tem em gerenciar ideias, o que inclui torná-las disponíveis para alimentar o processo de desenvolvimento de produtos.

Do ponto de vista da inovação como processo, este pode estar dividido em três subprocessos (SMITH; REINERTSEN, 1991; KOEN et al. 2001). O primeiro traz a etapa da identificação de oportunidades, geração ideia, e a definição de conceitos. O segundo e o terceiro, correspondem, respectivamente, ao desenvolvimento, e comercialização/lançamento ou implementação (SMITH; REINERTSEN, 1991; COOPER, 1988; KOEN et al., 2001; KOEN, BERTELS, KLEINSCHMIDT, 2014). As duas últimas etapas, embora em contínua melhoria, já estão bem estruturadas e possuem resultados esperados. Diferentemente da etapa inicial é onde as atividades são mais difíceis de definir e por consequência de gerenciar, pois apresentam grau elevado de incertezas (KOEN et al., 2001; KOEN; BERTELS; KLEINSCHMIDT, 2014).

Esta fase inicial é denominada por Koen et al. (2001) como Front End da Inovação (FEI), o qual envolve as atividades que ocorrem antes do processo de desenvolvimento de novos produtos. Em seu modelo para o FEl, Koen et al. (2002), apresenta como um dos elementos centrais as ideias, além de oportunidades e conceitos. Neste modelo as ideias são geradas, enriquecidas para posteriormente serem selecionadas.

Para Sandstrom e Bjork (2010) as empresas estão repensando como elas geram ideias e as trazem para o mercado. Ao utilizarem o paradigma da inovação aberta, difundido por Chesbrough (2006) envolvem o conhecimento e ideias de clientes, fornecedores e toda a comunidade. Xie e Zhang (2009) argumentam que, quando bem aplicado um método de geração de ideias, principalmente quando envolve a colaboração e concursos de ideias via

Perspectivas em Gestão \& Conhecimento, João Pessoa, v. 10, n. 3, p. 205-225, set./dez. 2020. 
web, pode-se facilmente gerar centenas delas. Torna-se uma questão complexa gerenciar esse fluxo, pois são muitos dados dentro de um ambiente que falta informação para tomada de decisão efetiva. Como por exemplo para a decisão de quais ideias seguirão adiante.

A preocupação em trabalhar com as ideias e gerenciá-la existe, desde o surgimento das primeiras "caixas de sugestões" nas organizações (WESTERSKI; IGLESIAS NAGLE, 2011). No entanto, atualmente surge a necessidade de otimização deste gerenciamento mediante a natureza da origem, complexidade e quantidade dessas ideias.

A literatura apresenta trabalhos que buscam criar modelos, processos ou guias para tal fim, uma vez que é necessário um processo definido para que haja a Gestão de Ideias (GI) (AAGAARD, 2013, NAGGAR, 2015). No entanto os modelos encontrados na literatura partem de diferentes focos e com diferentes entendimentos do que trata gerir ideias, como exemplo os trabalhos de Warkseski, Inglessias e Perez (2011) e Bothos, Apostolou e Mentzas (2009) apresentam modelos para a construção de Sistemas de Gestão de Ideias (SGI).

Dada a existência desta corrente que busca a estruturação da gestão de ideias (modelos e sistemas) e a importância desta para a organização, a pesquisa objetiva identificar os elementos que caracterizam o processo de gestão de ideias com foco no front end da inovação.

O trabalho está organizado em cinco seções a contar com esta introdução. A segunda seção traz o contexto da gestão de ideias e definições, a terceira seção apresenta a metodologia que se baseou em uma revisão integrativa. Na quarta seção são apresentados os elementos encontrados que compõem a gestão de ideias e por fim, na quinta seção são feitas as considerações finais.

\section{GESTÃO DE IDEIAS}

Diante de um mercado competitivo como o atual, onde é necessário que as empresas estejam preparadas para novos desafios, o termo inovação está consolidado como premissa básica para sobrevivência. Bessant et al. (2005) apontam que a habilidade das empresas em produzir inovações necessita de uma demanda contínua de ideias. Sukhov (2018) completa descrevendo que encontrar novas ideias é o primeiro passo para a condução de inovações em produtos ou serviços.

Neste contexto a Gestão de Ideias torna-se fator importante, principalmente devido ao fato que as ideias devem ser classificadas conforme a aderência aos interesses da empresa. Björk et al (2010) afirma que a gestão de ideias é parte fundamental do processo inovativo e pode significar o início deste processo, identificando oportunidades viáveis para as empresas. Conforme explica Gambatese e Hallowell (2011) diversos componentes e atividades estão relacionadas a gerar novas Ideias, e estes são parte do processo de inovação. Para Voight e Brem (2006) muitas empresas usam instrumentos de gestão de ideias para gerar inovações em seus ambientes, destacando que ideias são consideradas como a principal matéria-prima do processo de inovação.

Para Mileskone e Liesla (2015) a primeira menção sobre o termo gestão de ideias foi em Galbraith (1982) o qual o tratou como um processo cognitivo e social, enquanto a segunda menção foi de Green, Bean e Snavely (1983) o qual trataram a gestão de ideias numa perspectiva estrutural. Os autores analisaram a gestão de ideias como análoga ao processamento da informação humana e as implicações da tecnologia sobre este processo que inclui a geração, captura, retenção e recuperação de ideias.

Já para Voight e Brem (2006) a primeira definição sobre o termo foi feita por Siegfried Spahl em 1975 no livro "Handbuch Vorschlagswesen". Este autor classificou a gestão de ideias como um sistema que se dedica ao uso de métodos de criatividade e coleta de ideias em determinado tempo com relação próxima aos sistemas de sugestões.

Perspectivas em Gestão \& Conhecimento, João Pessoa, v. 10, n. 3, p. 205-225, set./dez. 2020. 
Independentemente de seu início, Jensen (2012) estabelece duas perspectivas para analisar a gestão de ideias: a comportamental e a estrutural.

Na perspectiva comportamental o foco é a compreensão da cognição, criatividade e capital social (JENSEN, 2012). Segundo a autora, nessa perspectiva o termo gestão de ideias é utilizado a nível de indivíduo ou visto como um processo social. Isto inclui preocupações de como as ideias são desenvolvidas e promovidas por meio de negociação na organização (GALBRAITH, 1982, JENSEN, 2012).

Já na perspectiva estrutural, o foco é na construção de sistemas e projetos para gerenciar ideias. Nesta perspectiva, a literatura baseia-se principalmente no campo da gestão da inovação e no desenvolvimento da tecnologia da informação com aplicações de sistemas de gerenciamento de ideias (XIE \& ZHANG, 2009, WESTERSKI; INGLESIAS; NAGLE, 2011, ALESSI et al., 2015). Estes estudos implicam, na maioria das vezes, um sistema de Tecnologias da Informação (TI) de compartilhamento e armazenamento de dados.

Jensen (2012) e Mileskone e Leisla (2015) concluem que ambas as perspectivas (comportamental e estrutural) são apropriadas, porque a gestão de ideias pode ser caracterizada não só como processo organizacional, mas também como um processo individual e social. $O$ entendimento destas duas visões é relevante porque o gerenciamento de ideias está fortemente relacionado a estruturação de processo e ao uso de sistemas de captura, compartilhamento, armazenamento e recuperação de ideias, mas ainda é considerado um processo social complexo que envolve interações tecnológicas e humanas (JENSEN, 2012; MILESKONE; LEISLA, 2015). Portanto neste trabalho reconhece-se a necessidade da interação entre as duas perspectivas.

O termo gestão de ideias está relacionado a outras atividades e conceitos além da inovação (MILESKONE; LEISLA, 2015). Como por exemplo, a gestão do conhecimento, business intelligence e aspectos que ocorrem dentro e fora das organizações (Inovação Aberta). Para compreender a iteração entre os conceitos a Figura 1 traça uma evolução do termo gestão de ideias ao longo dos anos com base nos autores pesquisados.

Figura 1: Evolução do termo gestão de ideias

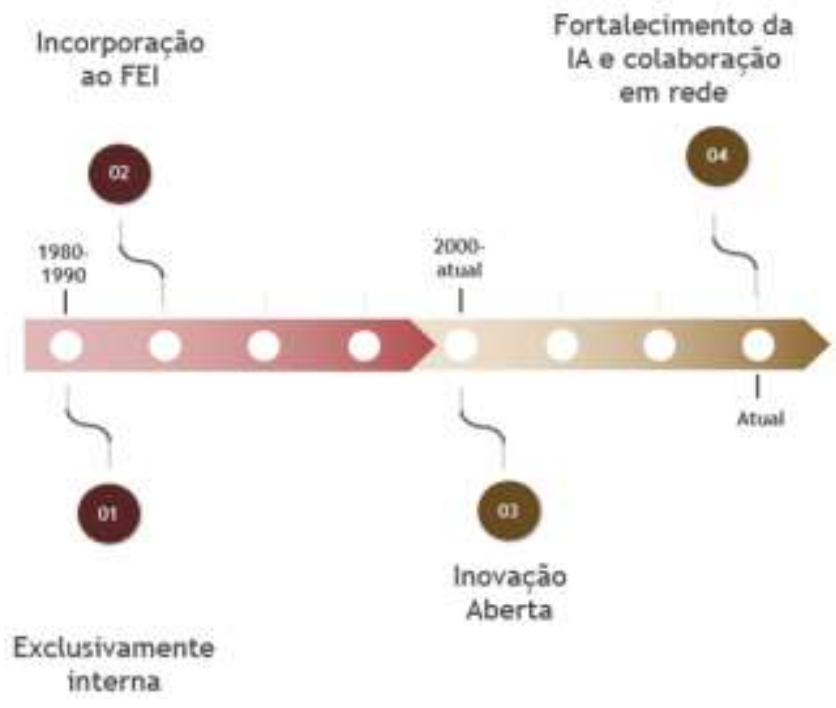

Fonte: Brem e Voigt (2009), Bothos, Apostolou, Mentza (2012), Aagaard (2013) e Perez et al. (2014)

Inicialmente, o principal objetivo da gestão de ideias era capturar as ideias dos funcionários, para uso posterior e possível desenvolvimento (GLASSMAN, 2009). Estava relacionada aos movimentos de melhorias contínua dos anos 1980 e 1990. Na sequência,

Perspectivas em Gestão \& Conhecimento, João Pessoa, v. 10, n. 3, p. 205-225, set./dez. 2020. 
muitas publicações na área relacionando a gestão de ideias com inovação, principalmente a incorporando ao Front end da inovação sob uma perspectiva gerencial (Koen et al. 2001, Brem, Voigt, 2009). Neste sentido, para Jensen (2012) a gestão de ideias é o reconhecimento de um processo criativo que pode ser gerenciado a fim de reduzir as incertezas no Front End da Inovação objetivando aumentar a capacidade de inovação

Paralelamente ao surgimento dos movimentos de inovação aberta estenderam essa definição para incluir a captura de ideias de entidades externas como clientes, fornecedores, inventores, universidades ou laboratórios nacionais (BOTHOS, APOSTOLOU, MENTZA, 2012, AAGAARD, 2013, ALESSI et al., 2015, GERLACH; BREM, 2017). Deste modo, o compartilhamento e armazenamento de dados, informações e conhecimentos podem ir além da organização. Este fato é resultante da evolução da gestão de ideias, bem como dos modelos de inovação que culminou a integração do ambiente interno ao externo da organização (BREM, VOIGT, 2009, BOTHOS, APOSTOLOU, MENTZA, 2012, AAGAARD, 2013, ALESSI et al. 2015 E BARRIOS et al. 2018).

Entender as diferentes perspectivas da gestão de ideias, bem como a evolução do conceito, auxilia no entendimento dela como um processo dentro da organização. Pois, envolve a interação entre os processos e sistemas explícitos e as práticas diárias de trabalho. Deste modo, a formalização do processo de ideias é importante para a organização, mas não deve ser o único objetivo da gestão de ideias e sim, ser entendido como um caminho para facilitar suas práticas (JENSEN, 2012).

Além disso, a visão complementar de que esse processo, pode ser entendido também na forma de um ciclo de vida é fundamental para ressaltar as interações, tanto de atores quanto de informação e conhecimento. Uma vez que neste ciclo, diferentes atores tanto internos quanto externos trocam conhecimento entre si (IVERSEN et al., 2009, WESTERSKI et al., 2011).

Outro elemento que auxilia no entendimento da gestão de ideias é sua delimitação a nível organizacional, pois algumas definições envolvem atividades que vão além do Front End da Inovação, como a implementação e a implantação da ideia. Para Koen et al. (2014) nestas atividades elas já passaram de ideias para um conceito ou produto de fato. Westerski et al. (2011) apesar de preverem estas etapas em seu modelo de sistema de gestão de ideias, ressaltam que elas já possuem processos estabelecidos e outros métodos e sistemas que os gerenciem. Não cabe a gestão de ideias competir com essas formas, mas sim auxiliar fornecendo um status e ter como foco o Front End.

Por isso, este trabalho nos próximos tópicos apresenta a caracterização do processo de gestão de ideias com foco no front end da inovação e aprofunda na descrição dos elementos, os quais inclui as fases/etapas, atores e funções que o compõem.

\section{PROCEDIMENTOS METODOLÓGICOS}

A fim de identificar os elementos na literatura de gestão de ideias, utilizou-se como procedimento metodológico uma revisão integrativa da literatura realizada por pares a qual seguiu os passos baseados nas indicações feitas por Torraco (2005) e Souza, Silva e Carvalho (2010). Foram eles:

a) Escolha do tema e definição da pergunta de pesquisa: O tema trata-se da "Gestão de ideias" e a pergunta pesquisa é: Quais os elementos, com base na literatura, caracterizam o processo de gestão de ideias nas organizações?

b) Escolha das bases de dados: As bases utilizadas foram Scopus, por ser uma base interdisciplinar e estar condizente com este estudo e a Web Of Science (WOS) por sua reconhecida qualidade de trabalhos.

Perspectivas em Gestão \& Conhecimento, João Pessoa, v. 10, n. 3, p. 205-225, set./dez. 2020. 
c) Estratégia de busca: A estratégia de busca utilizou-se dos descritores "idea management" OR "idea managing", com limitação para artigos em revistas e eventos. A intenção foi abranger artigos que possuíssem revisões por pares. Essas buscas retornaram 139 registros na Scopus e 59 na WOS, desse modo ao todo foram 198 registros buscados em outubro de 2017 e atualizados em maio de 2019.

d) Verificação dos duplicados e busca por artigos completos: Após a verificação de duplicados e a busca por artigos completos restaram 106. Nesta etapa utilizou-se o gerenciador de bibliografia Endnote como ferramenta de apoio.

e) Critérios de eleição: Para serem aceitos no portfólio final os artigos deveriam apresentar alguma forma para estruturar a gestão de ideias, ou seja, elementos, modelos, frameworks, processos, sistemas ou etapas claras. A clareza das etapas e a descrição destes elementos foi considerado critério importante para a escolha. Portanto, não bastava apenas mencionar o elemento e sim descrevê-lo.

f) Leitura e composição do portfólio: Os 106 artigos foram lidos seus títulos, resumos e palavras-chave e foram eliminados os que não apresentavam os critérios mencionados. Após essa análise restaram 48 documentos. Esses foram lidos na íntegra e foram selecionados 16 documentos que detalharam com clareza 0 processo, framework ou modelo de gestão de ideias. O Quadro 1 reúne os artigos do portfólio.

Quadro 1: Portfólio de artigos para análise

\begin{tabular}{|c|c|}
\hline \multirow{2}{*}{ Autor/Ano } & \multirow{3}{*}{$\begin{array}{c}\text { Título } \\
\text { Idea management in } \mathrm{R} \& \mathrm{D} \text { as a human information processing analog }\end{array}$} \\
\hline & \\
\hline $\begin{array}{l}\text { Green, Bean e Snavely } \\
(1983)\end{array}$ & \\
\hline Voigt e Brem (2006) & Integrated idea management in emerging technology Ventures \\
\hline $\begin{array}{l}\text { Bothos, Apostolou e } \\
\text { Mentzas (2009) }\end{array}$ & $\begin{array}{l}\text { Collective intelligence for idea management with internet-based } \\
\text { information aggregation markets }\end{array}$ \\
\hline Sandström e Björk (2010) & Idea management systems for a changing innovation landscape \\
\hline Sint et al. (2010) & $\begin{array}{l}\text { Ideator - A collaborative enterprise idea management tool powered by } \\
\text { KiWi? }\end{array}$ \\
\hline Duin et al (2010) & An idea model for distributed Idea Management \\
\hline $\begin{array}{l}\text { Westerski, Iglesias e Nagle } \\
\text { (2011) }\end{array}$ & $\begin{array}{l}\text { The road from community ideas to organisational innovation: A life } \\
\text { cycle survey of idea management systems }\end{array}$ \\
\hline $\begin{array}{l}\text { Stevanovic, Marjanovic e } \\
\text { Storga (2013) }\end{array}$ & Idea relevancy assessment in preparation of product development \\
\hline Aagaard (2013) & $\begin{array}{l}\text { A theoretical model of supporting open source front end innovation } \\
\text { through idea management }\end{array}$ \\
\hline Murah et al. (2013) & Kacang cerdik: A conceptual design of an idea management system \\
\hline El Bassiti e Ajhoun (2014) & Semantic-Based Framework for Innovation Management \\
\hline $\begin{array}{l}\text { Perez, Larrinaga e Curry } \\
\text { (2014) }\end{array}$ & $\begin{array}{l}\text { The role of linked data and semantic-technologies for sustainability idea } \\
\text { management }\end{array}$ \\
\hline Alessi et al. (2015) & $\begin{array}{l}\text { Applying Idea Management System (IMS) approach to design and } \\
\text { implement a collaborative environment in public service related open } \\
\text { innovation processes }\end{array}$ \\
\hline Gallmeister e Lutz (2016) & Engagement and retention: Essentials of idea management \\
\hline Gerlach e Breim (2017) & $\begin{array}{l}\text { Idea management revisited: A review of the literature and guide for } \\
\text { implementation }\end{array}$ \\
\hline
\end{tabular}

Fonte: Elaborado pelos autores com base na literatura revisada

Perspectivas em Gestão \& Conhecimento, João Pessoa, v. 10, n. 3, p. 205-225, set./dez. 2020. 
g) Análise do portfólio: Para identificar os elementos que compõe o processo de gestão de ideias, os artigos selecionados no portfólio foram analisados e deles isolados cada elemento. Após eles foram agrupados por similaridade semântica, assim como a frequência que apareciam.

\section{RESULTADOS E DISCUSSÕES}

Com a análise do portfólio (Quadro 1) constatou-se que parte dos modelos encontrados para gestão de ideias são baseados em modelos para a construção de sistemas. Isso se deve, como argumenta Westerski, Iglesias e Nagle (2011) e Sandström e Björk (2010) que parte da área evolui com base na criação destes sistemas, iniciando como sistemas de sugestões incorporados em sistemas corporativos, até os modelos mais atuais envolvendo tecnologia semântica e colaborativa.

Dos modelos para sistemas de gestão de ideias (SGI), pode-se dividir em dois grupos: 1) com foco na utilização de tecnologia semântica, como em: Westerski, Iglesias e Nagle (2011), El Bassiti e Ajhoun (2014), Perez, Larrinaga e Curry (2014)); 2) com foco na colaboração, como em: Bothos, Apostolou e Mentzas (2009), Sint et al. (2010), Murah et al. (2013) e Alessi et al. (2015).

Ainda, Sandström e Björk (2010) analisam a inserção de um SGI em uma organização na qual fazem tratamento diferente entre ideias com potencial para inovação disruptiva e incrementais. Duin et al (2010) propõem a construção de um sistema par armazenamento distribuído de ideias. Por fim, destaca-se Green, Bean e Snavely (1983) como o primeiro artigo encontrado nas bases que explicita o processo de Gestão de Ideias fazendo a analogia com processamento da informação humana. Apesar dos autores não mencionarem SGI, eles fazem forte alusão ao uso de sistemas e tecnologias apoiadoras.

Neste contexto, os SGI atuam como ferramentas que dão apoio e facilitam as fases presentes na gestão de ideias. Eles são softwares ou aplicativos que fornecem repositórios e ferramentas para coletar, procurar, editar, comentar e votar ideias (PEREZ, LARRINAGA, CURRY, 2014). Ainda segundo os autores eles podem conter diferentes concursos ou campanhas de ideias, bem como stakeholders (atores). Eles geralmente estão vinculados ao conceito de inovação aberta no ambiente Web com o uso de técnicas como o crowdsourcing (WESTERSKI, IGLESIAS, NAGLE, 2011)

Voigt e Brem (2006), Stevanovic, Marjanovic e Storga (2013), Aagaard (2013), Gallmeister e Lutz (2016) e Gerlach e Breim (2017), adotaram uma abordagem que vai além dos sistemas de gestão de ideias como a apoio a gestão. Estes autores constroem modelos ou frameworks contendo as fases que podem ser apoiadas pelos referidos sistemas, mas também políticas, tecnologias e o ambiente organizacional propício para que aconteça a gestão de ideias. Por exemplo em Aagaard (2013), Gallmeister e Lutz (2016) e Gerlach e Breim (2017) apresentam fases, na qual inicia com uma preparação antes de começar a geração de ideias propriamente dita. Já Stevanovic, Marjanovic e Storga (2013) foca na avaliação e seleção de ideias.

Algumas fases para a gestão de ideias já estão consolidadas, como geração, melhoramento, avaliação e seleção. No entanto, buscou-se identificar possíveis ligações e elementos padrões que as compõem. Além disso, permitiu-se verificar que são usadas diferentes terminologias as quais foram mapeadas, agrupadas por semelhança e dispostas no Quadro 2, com os respectivos autores que as citam. Faz-se notar também que o armazenamento e feedback foram consideradas fases que perpassam pelas demais.

Perspectivas em Gestão \& Conhecimento, João Pessoa, v. 10, n. 3, p. 205-225, set./dez. 2020. 
Quadro 2: Fases e suas terminologias encontradas na literatura

\begin{tabular}{|c|c|c|}
\hline Fases /etapas & Autores & Definição \\
\hline Preparação & $\begin{array}{l}\text { Aagaard (2013), Vagn, Clausen e } \\
\text { Gish (2013), Gallmeister e Lutz } \\
\text { (2016), Gerlach e Breim (2017). }\end{array}$ & $\begin{array}{l}\text { Primeira fase do processo, responsável pela } \\
\text { definição das diretrizes do programa, bem } \\
\text { como preparação do ambiente e pessoas. }\end{array}$ \\
\hline Geração de Ideias & $\begin{array}{l}\text { Green, Bean e Snavely (1983), } \\
\text { Bothos, Apostolou e Mentzas } \\
\text { (2009); Westerski, Iglesias e Nagle } \\
\text { (2011), Aagaard (2013), Stevanovic, } \\
\text { Marjanovic e Storga (2013), Vagn, } \\
\text { Clausen e Gish (2013), El Bassiti e } \\
\text { Ajhoun (2014), Perez e Laringana } \\
\text { (2014), Alessi et al (2015), } \\
\text { Gallmeister e Lutz (2016), Gerlach e } \\
\text { Breim (2017). }\end{array}$ & $\begin{array}{l}\text { Processo ativo, apoiado fortemente por } \\
\text { técnicas criativas. Tem o objetivo de fornecer } \\
\text { entradas ao programa. Ideias podem ser } \\
\text { geradas a partir de problemas e-ou } \\
\text { oportunidades. Deve ser investido na } \\
\text { capacitação do indivíduo para posteriormente, } \\
\text { trabalhar-se com grupos criativos. }\end{array}$ \\
\hline Coleta de Ideias & $\begin{array}{l}\text { Green, Bean e. Snavely (1983), } \\
\text { Voigt e Brem (2006), Sandström e } \\
\text { Björk, (2010), Aagaard (2013), } \\
\text { Gallmeister e Lutz (2016). }\end{array}$ & $\begin{array}{l}\text { Processo passivo, apoiado fortemente por } \\
\text { mecanismos que forneçam meios de recolha } \\
\text { das ideias. Normalmente feitos por formulários } \\
\text { online ou físicos. }\end{array}$ \\
\hline Filtragem de ideias & $\begin{array}{l}\text { Sandström e Björk } \\
\text { Gallmeister e Lutz (2016). }\end{array}$ & $\begin{array}{l}\text { Responsável pela verificação de ideias } \\
\text { duplicadas ou similares. Objetiva reduzir o } \\
\text { portfólio inicial. }\end{array}$ \\
\hline Categorização & Sandström e Björk (2010). & $\begin{array}{l}\text { Responsável por agrupar ideias por temáticas. } \\
\text { Partindo de categorias pré-fornecidas ou não. } \\
\text { Objetiva buscar sentido por meio de grupos } \\
\text { temáticos. }\end{array}$ \\
\hline $\begin{array}{l}\text { Enriquecimento de } \\
\text { ideias }\end{array}$ & $\begin{array}{l}\text { Voigt e Brem (2006), Bothos, } \\
\text { Apostolou e Mentzas (2009), } \\
\text { Westerski, Iglesias e Nagle (2011), } \\
\text { Stevanovic, Marjanovic e Storga } \\
\text { (2013), Muhah et al (2013), El } \\
\text { Bassiti e Ajhoun (2014), Perez e } \\
\text { Laringana (2014), Alessi et al (2015), } \\
\text { Gallmeister e Lutz (2016), Gerlach e } \\
\text { Breim (2017). }\end{array}$ & $\begin{array}{l}\text { Processo essencialmente colaborativo, no qual } \\
\text { as ideias previamente sugeridas são } \\
\text { trabalhadas e reformuladas, por meio de } \\
\text { técnicas criativas e colaborativas. O objetivo é } \\
\text { trazer novas informações e conhecimentos as } \\
\text { ideias iniciais. Neste momento, novas ideias } \\
\text { podem serem criadas a partir das existentes ou } \\
\text { recombinadas por meio dos grupos e } \\
\text { categorias identificados. }\end{array}$ \\
\hline Avaliação de ideias & $\begin{array}{l}\text { Voigt e Brem (2006), Bothos, } \\
\text { Apostolou e Mentzas (2009), } \\
\text { Aagaard (2013), Stevanovic (2013), } \\
\text { Vagn, Clausen e Gish (2013), Muhah } \\
\text { et al (2013), El Bassiti e Ajhoun } \\
\text { (2014) }\end{array}$ & $\begin{array}{l}\text { Processo analítico feito por meio de critérios } \\
\text { previamente estabelecidos, não objetiva a } \\
\text { eliminação das ideias, mas sim oportunizar o } \\
\text { aprimoramento e dá suporte a tomada de } \\
\text { decisão. }\end{array}$ \\
\hline Seleção de ideias & $\begin{array}{l}\text { Sandström e Björk (2010), } \\
\text { Westerski, Iglesias e Nagle (2011), } \\
\text { Aagaard (2013), Stevanovic (2013), } \\
\text { Vagn, Clausen e Gish (2013), Perez } \\
\text { e Laringana (2014), Alessi et al } \\
\text { (2015) }\end{array}$ & $\begin{array}{l}\text { Processo de tomada de decisão, escolha das } \\
\text { ideias após os melhoramentos. Baseia-se em } \\
\text { critérios e métodos de tomada de decisão. }\end{array}$ \\
\hline $\begin{array}{l}\text { Priorização } \\
\text { ideias }\end{array}$ & Gallmeister e Lutz (2016) & $\begin{array}{l}\text { Criação de um ranking das ideias selecionadas } \\
\text { por meio de métodos definidos. }\end{array}$ \\
\hline $\begin{array}{l}\text { Refinamento das } \\
\text { ideias }\end{array}$ & $\begin{array}{l}\text { Sandström e Björk (2010), Aagaard } \\
\text { (2013), Alessi et al (2015) }\end{array}$ & $\begin{array}{l}\text { Feito após as ideias serem selecionadas, busca- } \\
\text { se informações para acrescer na sua definição } \\
\text { e posteriormente tornar-se um conceito de }\end{array}$ \\
\hline
\end{tabular}

Perspectivas em Gestão \& Conhecimento, João Pessoa, v. 10, n. 3, p. 205-225, set./dez. 2020. 


\begin{tabular}{|c|c|c|}
\hline Fases /etapas & Autores & Definição \\
\hline & & produto. \\
\hline Armazenamento & $\begin{array}{l}\text { Green, Bean e Snavely (1983), } \\
\text { Spancer (2007), Duin et al (2010), } \\
\text { Sandström e Björk (2010), } \\
\text { Westerski, Iglesias e Nagle (2011), } \\
\text { Gallmeister e Lutz, (2016) }\end{array}$ & $\begin{array}{l}\text { É apoio para todas as demais fases } \\
\text { identificadas armazenando as ideias e as } \\
\text { informações e sobre essas ideais. }\end{array}$ \\
\hline $\begin{array}{l}\text { Feedback e } \\
\text { acompanhamento }\end{array}$ & $\begin{array}{l}\text { Westerski, Iglesias e Nagle (2011), } \\
\text { Alessi et al (2015), Gallmeister e } \\
\text { Lutz, (2016). }\end{array}$ & $\begin{array}{l}\text { Perpassa por todas as demais fases } \\
\text { identificadas, baseando-se em métricas para o } \\
\text { controle do programa, bem como retorno das } \\
\text { atividades feitas. }\end{array}$ \\
\hline
\end{tabular}

Fonte: Elaborado pelos autores com base na literatura revisada

Foram mapeadas também as funções que estão presentes em cada fase. 0 Quadro 3 apresenta as funções, bem como os autores que as citam e a definição construída com base nestes autores. Faz-se esclarecer que os atores durante o processo podem ser usuários, colaboradores, fornecedores, consumidores, a comunidade e outras partes interessadas como a academia ou pesquisadores. Estes atores ou usuários podem assumir múltiplos papéis (MURAH et al., 2013). Por exemplo, funcionários, gestores, consumidores, podem assumir papel de "Ideador". Além disso, atores podem ser humanos e não humanos (VAGN, CLAUSEN e GISH, 2013).

Quadro 3: Funções e atores que compõem a gestão de ideias

\begin{tabular}{|c|c|c|}
\hline Função & Autores & Definição \\
\hline $\begin{array}{lr}\text { Gestor } & \text { de } \\
\text { ideias } & \text { e } \\
\text { comitê } & \text { de } \\
\text { gestão } & \text { de } \\
\text { ideias } & \end{array}$ & $\begin{array}{l}\text { Bothos, Apostolou e } \text { Mentzas } \\
\text { (2009), Sint et al. (2010), } \\
\text { Sandström e Björk (2010) Perez, } \\
\text { Larrinaga e Curry (2015). }\end{array}$ & $\begin{array}{l}\text { O gestor da iniciativa de Gestão de ideias } \\
\text { recomenda-se função exclusiva. Pode formar um } \\
\text { comitê de gestão de ideias, ou seja, pode chamar a } \\
\text { ajuda de pessoas externas para compor o comitê } \\
\text { que irá auxiliar na política de funcionamento do } \\
\text { programa bem como no monitoramento. }\end{array}$ \\
\hline Ideador & $\begin{array}{l}\text { Green, Bem e Snavely, (1883), } \\
\text { Voigt e Brem (2006), Bothos, } \\
\text { Apostolou e Mentzas (2009), Sint, } \\
\text { Markus, Schaffert e Kurz (2010), } \\
\text { Muhah et al (2013), Perez, } \\
\text { Larrinaga e Curry (2015), Alessi et } \\
\text { al (2015), Gallmeister e Lutz (2016) }\end{array}$ & $\begin{array}{l}\text { Função responsável por fornecer a entrada para o } \\
\text { programa de GI. Diferentes atores podem assumir a } \\
\text { função de ideador: colaboradores, outras partes } \\
\text { interessadas, funcionários, consumidores, } \\
\text { fornecedores e a comunidade em geral. }\end{array}$ \\
\hline Especialista & $\begin{array}{l}\text { Bothos, Apostolou e Mentzas } \\
\text { (2009). }\end{array}$ & $\begin{array}{l}\text { Os especialistas possuem conhecimento de domínio } \\
\text { requerido aquela ideia. Eles podem ser internos ou } \\
\text { externos à organização. }\end{array}$ \\
\hline Colaborador & Muhah et al. (2013) & $\begin{array}{l}\text { Função assumida por aqueles que irão enriquecer e } \\
\text { melhorar uma ideia por meio de um processo de } \\
\text { colaboração. }\end{array}$ \\
\hline Avaliador & Muhah et al. (2013). & $\begin{array}{l}\text { Função que antecede a dos tomadores de decisão. } \\
\text { Atribui a questão de votação de ideias, o qual está } \\
\text { relacionado ao conceito de sabedoria das } \\
\text { multidões. }\end{array}$ \\
\hline $\begin{array}{l}\text { Tomadores } \\
\text { de decisão }\end{array}$ & $\begin{array}{l}\text { Bothos, Apostolou e Mentzas } \\
\text { (2009), Sint et al. (2010), Muhah et } \\
\text { al (2013), Perez, Larrinaga e Curry } \\
\text { (2015). }\end{array}$ & $\begin{array}{l}\text { São os responsáveis por tomar as decisões durante } \\
\text { o processo. Normalmente são solicitados na fase de } \\
\text { avaliação, seleção e priorização das ideias. } \\
\text { Normalmente são gestores com nível hierárquico } \\
\text { mais alto, mas, podem incluir especialistas de } \\
\text { domínio e a comunidade tanto interna quanto }\end{array}$ \\
\hline
\end{tabular}

Perspectivas em Gestão \& Conhecimento, João Pessoa, v. 10, n. 3, p. 205-225, set./dez. 2020. 


\begin{tabular}{|l|l|c|}
\hline Função & Autores & Definição \\
\hline & externa para auxílio na tomada de decisão \\
\hline
\end{tabular}

Fonte: Elaborado pelos atores com base na literatura estudada

A seguir as fases identificadas, bem como os demais elementos e atores são descritas em detalhes.

\subsection{Preparação}

A fase de preparação é responsável pelo estabelecimento de políticas de funcionamento das iniciativas de gestão de ideias. Gerlach e Breim (2017) descrevem que nesta etapa devem ser definidas as normas para a participação dos colaboradores da organização, bem como a definição de um nome que permita que os participantes façam a associação correta com o objetivo da ação. Logo, deve estar apoiado por uma divulgação concisa, para chamar atenção dos possíveis participantes (GERLACH; BREIM, 2017). O esclarecimento do que é uma ideia, ou seja, campos que a ideia deve ter na hora da submissão pode agregar valor ao programa (GALLMAISTER; LUTZ, 2016). Contudo, estas características, não podem limitar ou inibir a criatividade dos participantes.

A organização pode optar por implantar um programa de gestão de ideias baseado em desafios. Para isso, é necessário mapear quais desafios ou problemas, traduzi-los para uma linguagem que possa ser entendida por todos os participantes e promover a sua divulgação (SPANCER, 2007; BOTHOS et al., 2012; GERLACH, BREIM 2017). Neste tipo de ação pode haver o envolvimento da comunidade externa e demais stakeholders. Aggard (2013) argumenta a necessidade de realizar uma varredura e mapeamento de conhecimento, com o objetivo de motivar os colaboradores para pesquisar sistematicamente em uma base regular em que estão inseridos clientes, tecnologia e outros. Gerlach e Breim (2017) acrescentam que para gerenciar todo este processo um gestor deve ser designado, recomenda-se, no entanto, que, este gestor seja específico para comandar todo o processo.

Desta forma, o principal ator desta fase é o gestor de ideias, sendo este responsável pela gestão da iniciativa (SINT et al., 2010; BOTHOS, APOSTOLOU, MENTZAS, 2009; PEREZ, LARRINAGA; CURRY, 2015) e por formar o comitê de gestão de ideias (SANDSTROM, BOJORK, 2010) que inclui todos os atores internos e externos que irão atuar durante as fases do programa.

A próxima etapa, geração de ideias, é influenciada significativamente pela forma como as empresas pesquisam, digitalizam, geram ou obtém informações para ideias. Uma vez que ideias são provenientes de conhecimentos e experiências, que são detidos pelos funcionários, incluindo novos conhecimentos adquiridos internamente e externamente (AGGARD, 2013). A decisão de utilizar de softwares para auxiliar o processo, como os sistemas de gestão de ideias devem ser previstos nesta etapa. Como resultado principal desta etapa têm-se as políticas de funcionamento das iniciativas de gestão de ideias.

\subsection{Geração e coleta de ideias}

$\mathrm{Na}$ fase anterior o principal objetivo era fornecer conhecimento e inspiração para ideias, desse modo, a fase de geração e coleta de ideias deve se concentrar no desenvolvimento de várias ideias através destes conhecimentos e inspirações, que juntas formam o que a organização está procurando. Ideias e problemas estão intimamente interligados e, em muitos casos, não são separáveis. O mesmo acontece com ideias e oportunidades, no qual o reconhecimento ou a criação de uma oportunidade é uma ocasião para geração de ideias, bem como, uma ideia pode levar a uma oportunidade (AGGARD, 2013).

Perspectivas em Gestão \& Conhecimento, João Pessoa, v. 10, n. 3, p. 205-225, set./dez. 2020. 
Para Gallmeister e Lutz (2016) ideias são geradas e coletadas de modos diferentes utilizando técnicas distintas, como por exemplo, técnicas de brainstorming, análise de problemas, análise de mapa perceptual, grupos de foco no cliente, mapeamento de produtos concorrentes, melhorias do produto, desenvolvimentos tecnológicos, pesquisa e troca de informações. Os resultados da aplicação de tais técnicas são apenas fragmentos de ideia que precisa ser mais explorado (GALLMAISTER; LUTZ, 2016).

Tanto a geração quanto a coleta são responsáveis pelas entradas de ideias do programa de gestão de ideias. No entanto, a geração de ideias é um processo ativo, no qual são criados conhecimentos, enquanto a coleta de ideias, é um processo passivo de reunir ideias já existentes dentro e fora da organização. Em algumas organizações, isto é designado por uma "caixa de sugestões", onde os funcionários colocam e armazenam suas ideias. Em outras empresas instrumentos formais diversificados ou sistemas de gerenciamento de ideias são integrados para reunir e identificar de forma contínua a geração e coleta de ideias (AGGARD, 2013).

Para a coleta de ideias sugere-se que as submissões sejam feitas usando um modelo, na forma de formulário tanto online quanto físico. Sandström e Björk (2010) sugerem os seguintes itens para formulários: Problema ou oportunidade que a ideia atende, os elementos chave, benefícios para os consumidores, benefícios para a companhia e possíveis dificuldades de desenvolvimento. Entende-se que este modelo de coleta de ideias deva ter sido definido na fase de preparação.

A criação de ideias de inovação e seu desenvolvimento são resultados da síntese de conhecimentos diversos e de reuniões imprevistas através de diferentes tipos de relações entre indivíduos, coisas e estruturas. Diferentes fontes de conhecimento estão reunidas interagindo umas com as outras resultando em um fluxo contínuo de negociações e processos de construção, e engajamento com a organização, a fim de promover ideias e apresentá-las a estruturas corporativas e procedimentos formais (VAGN, CLAUSEN, GISH, 2013).

A criatividade desempenha papel crucial nesta fase. A habilidade criativa está em cada indivíduo e deve ser desenvolvida pela gestão (AMABILE, 1998, FLYNN et al 2003), pois é ela quem favorece um conjunto de pensamentos criativos que impulsionam o desenvolvimento de ideias (EL BASSITI; AJHOUN, 2014). O trabalho em equipe estimula a criatividade e gera melhores ideias. No entanto, quando grupos trabalham primeiro individualmente e depois em conjunto, eles produzem ideias com maior qualidade, do que aqueles grupos que logo começam a trabalhar juntos (GIROTRA et al., 2010). Ainda segundo os autores, grupos com diferentes origens culturais e diferentes níveis hierárquicos tendem a ser mais criativos.

Gerlach e Breim (2017) descrevem que as fontes de criatividade e ideias estão relacionadas com a interação com cliente, pesquisas de mercado, ideias já compartilhadas nos setores, além de novas leis e regras que o mercado ou fornecedores encontram. Dessa maneira fontes internas e externas são consideradas como fonte de criatividade e ideias.

As fontes externas para novas ideias, estão relacionadas ao conceito de inovação aberta de Chesbrough (2014). Advém principalmente, da relação entre organização, consumidor ao realizar transferência de know-how que pode melhorar a comunicação e sugerir otimizações específicas do produto ou então na utilização de componentes específicos do fornecedor que melhorem custos e produtos (VOIGT; BREM, 2006).

Independente das ideias advirem internamente ou externamente, nesta fase elas sozinhas ainda não possuem potencial de desenvolvimento, pois, podem ser meras descrições (STEVANOVIĆ; MARJANOVIC; STORGA, 2013), por isso devem continuar a serem trabalhadas e desenvolvidas, por meio de processos criativos e colaborativos.

Os principais atores nesta fase são os chamados "ideadores" que tem a função de fornecer ideias ao programa. Estes podem incluir desde os próprios colaboradores da organização (GALLMEISTER LUTZ, 2016; GREEN, BEM, SNAVELY, 1883, VOIGT, BREM, 2006),

Perspectivas em Gestão \& Conhecimento, João Pessoa, v. 10, n. 3, p. 205-225, set./dez. 2020. 
fornecedores e consumidores (ALESSI et al., 2015, BOTHOS, APOSTOLOU, MENTZAS, 2009, PEREZ, LARRINAGA, CURRY, 2015, VOIGT, BREM, 2006), a comunidade (SINT et al., 2010, ALESSI et al., 2015; VOIGT, BREM 2006) e outros interessados (ALESSI et al., 2015; VOIGT, BREM, 2006).

Como resultado desta fase o programa de ideias tem um portfólio de ideias "cruas", ou seja, são descrições iniciais que irão ser co-criadas ao longo do processo. Estas devem estar disponíveis para que todos os envolvidos tenham acesso.

\subsection{Filtragem, agrupamento, categorização de ideias}

Esta atividade não é considerada uma fase na maioria dos autores estudados, mas um passo necessário, já que dependendo da técnica utilizada para geração e coleta, esta pode gerar muitas ideias. Pode tornar-se comum que ideias similares apareçam, ou então que ideias se repitam (GALLMEISTER; LUTZ, 2016). Antes de aplicarem-se técnicas criativas para aperfeiçoar as ideias é necessário categorizá-las por temática ou semelhança e filtrar as repetidas ou que não possuem informações suficientes para prosseguir (GALLMEISTER; LUTZ, 2016).

Duin et al. (2010) sugere que devam ser formadas árvores de ideias de forma hierárquica, onde as ideias são reunidas em grupos e subgrupos. Já para esta fase Sandström e Björk (2010) preveem uma "avaliação grosseira" como forma de filtrar ideias e depois classificá-las em categorias seguirem no processo. As categorias sugeridas por ele são: ideias radicais (fora da caixa) e ideias incrementais (dentro da caixa).

Os responsáveis e demais atores envolvidos nesta fase seriam os próprios integrantes do comitê de gestão de ideias. Além disso, esta fase pode ter forte apoio ferramental, como de um sistema de gestão de ideias, ou ferramentas que façam o agrupamento e a clusterização das ideias, como nos trabalhos de Westerski, Inglesias e Nagle (2011). Ao final desta fase temse, portanto, a formação de grupos de ideias.

\subsection{Enriquecimento de ideias}

As ideias geradas anteriormente são apenas fragmentos de ideia que ainda não foram totalmente formuladas. Para que se transformem em ideias de alto valor é necessário combinar fragmentos, identificar as lacunas e gerar novos fragmentos de ideia que irão se complementarem (GALLMEISTER; LUTZ, 2016). Ainda segundo os autores isso pode ocorrer por um processo de reforço e triagem que gera e realça as ideias.

O objetivo central desta fase é permitir que as pessoas colaborem entre si para melhorar as ideias (WESTERSKI; IGLESIAS; NAGLE, 2011) para que a ideia evolua (MUHAH et al, 2013). A colaboração pode ser auxiliada com a criação de pontos físicos para a cooperação entre funcionários (VOIGT; BREM, 2006), bem como, realizada de forma virtual, por meio de um Sistema de Gerenciamento de Ideias (SGI) ou demais ferramentas colaborativas.

Com relação aos $\mathrm{SGl}$, eles podem facilitar a colaboração com a utilização de comentários, imagens, links dentre outros recursos (ALESSI et al., 2015). O uso destes sistemas facilita o processo colaborativo por meio da sabedoria da multidão ou inteligência coletiva (MUHA et al., 2013).

Para Alessi et al. (2015) a partir de uma ou mais ideias iniciais um processo de cocriação, socialização e troca de experiência e conhecimento é desencadeado. As ideias podem ser disponibilizadas a toda a comunidade que colabora para transformá-la. Portanto, a comunidade, devidamente apoiada, pode melhorar ideias, explorar know-how e múltiplas perspectivas emergentes (ALESSI et al, 2015).

Perspectivas em Gestão \& Conhecimento, João Pessoa, v. 10, n. 3, p. 205-225, set./dez. 2020. 
Já no Sistema de Gestão de ideias de Bothos et al. (2009) em formato de mercado de ações, os participantes podem investir em uma ideia e, em seguida, contribuir, questionar aspectos, e sugerir melhorias ou mudanças de acordo com sua visão pessoal.

Com relação a formas físicas para enriquecimento de ideias, apesar de não identificado explicitamente na revisão, o "World Coffee" pode servir para enriquecer ideias. É uma técnica flexível, sensível que promove a interação entre as pessoas, através da qual elas são incentivadas a opinarem e construírem o conhecimento de forma coletiva e descobrirem novas oportunidades de ação conjunta (BROWN, 2007). Outro modo de enriquecimento, é realizado a partir de interações físicas utilizando painéis distribuídos em locais estratégicos na organização.

Os atores nesta fase se caracterizam principalmente por grupos de discussão como aponta Gerlach e Breim (2017). Estes grupos podem ser compostos por: clientes, comunidade, colaboradores internos, fornecedores e especialistas que assumam a função de ser um grupo colaborativo. Ao final desta fase a organização tem seu portfólio de ideias enriquecido, podendo este portfólio aumentar ou diminuir a quantidade de ideias.

\subsection{Avaliação, seleção, priorização de ideias}

A avaliação e seleção de ideias são realizadas como forma de reduzir riscos e recursos que serão empregados para o desenvolvimento futuro da ideia. Desta forma, assim como a fase de geração de ideias é criativa, a avaliação e seleção é analítica (AAGARD, 2013). Esta fase é um ponto crítico no processo, uma vez que tomadas de decisões em fases iniciais da inovação, são difíceis devido ao nível de incertezas (poucas informações).

No entanto, ela pode se tornar menos onerosa se as demais fases forem realizadas corretamente, ou seja, ter um processo formal de geração de ideias e estas ideias estarem organizadas e armazenadas com descrições básicas. Gallmeister e Lutz (2016), argumentam também que se os critérios para uma boa ideia foram claramente definidos, será menos trabalhoso avaliá-la e posteriormente selecioná-la.

Desta forma, faz-se necessário definir os critérios corretos com base em objetivos estabelecidos, os quais podem mudar, ou não, a cada nova seleção. Stevanović Marjanovic e Storga (2013) definem critério como sendo o que deve medir algo importante e ser independente, visto ser comum que nenhuma ideia seja boa em todos os âmbitos.

Os mesmos autores estabelecem alguns critérios e estes possuem uma escala que vai de 1 a 5. São eles:

1) Novidade: do produto, para o usuário, de produção e para o mercado.

2) Custo: de desenvolvimento, de produção, comercialização e posteriores promoções.

3) Benefícios: do produto para o usuário, para a produção, para o ambiente.

4) Risco: tecnológico, mercadológico, de negócio, econômico.

Sandström e Björk (2010) sugerem como critérios: avaliação do mercado, tecnologia e novidade da ideia em relação aos recursos e capacidades da empresa. Estes autores ressaltam que se deve utilizar critérios diferentes para ideias de inovação radical e incremental. Se forem empregados os critérios padrão para as ideias radicais corre-se o risco delas não se encaixarem nestes critérios, pois não se encaixam no modelo de negócio atual da organização. Este é um ponto crítico tratado pelos autores, uma vez que a forte sistematização, por meio de métodos rígidos e critérios, podem inibir as inovações radicais de uma organização. Por outro lado, a não utilização de método e critério algum, pode deixar passar ideias de potenciais produtos.

Além disso, nesta etapa do processo, os critérios quando bem definidos tem a vantagem de serem utilizados para melhorar uma ideia promissora em vez de eliminá-la de imediato (GALLMEISTER, LUTZ, 2016). Sendo assim, as avaliações podem ser consideradas como forma de enriquecer também o processo para que depois seja feita a seleção.

Perspectivas em Gestão \& Conhecimento, João Pessoa, v. 10, n. 3, p. 205-225, set./dez. 2020. 
Neste artigo entende-se que a avaliação e posterior seleção, pode ocorrer em dois estágios, uma avaliação externa e outra por um grupo de tomada de decisão que é interno. Novamente a inovação aberta entra em questão, principalmente quando se trata de um sistema de Gestão Ideias. Pois, este pode aproveitar o alto volume de dados enviado pelas multidões na escolha das melhores ideias (WESTERSKI, INGLESIAS, NAGLE, 2011).

Esses dados podem vir em formato de votação como apresenta Alessi et al (2015) no qual a partir desta votação gera-se uma classificação em forma de ranking. Este ranking aponta ideias com maior prioridade ou as que os usuários consideram melhores do que outras. Os indicadores utilizados para a avaliação são, por exemplo: o número de threads ou o índice de vitalidade, que expressa como a ideia permanece ativa ao longo do tempo. Além disso, é possível fazer uma análise indireta de ideias através da análise de sentimentos que permita identificar as questões particularmente importantes para o cidadão / usuário (ALESSI et al., 2015). Muhah et al. (2013) reforça a utilização da sabedoria da multidão para determinar a qualidade da ideia e auxiliar no processo de escolha.

Mesmo passando por esta avaliação da multidão, Alessi et al (2015) afirma que a saída desta fase é a seleção de ideias que ainda serão analisadas em detalhes, estudando a sustentabilidade delas por meio de um comitê de seleção e por meio de critérios e métodos previamente definidos.

O comitê de tomada de decisão, pode ser formado por: Especialistas Internos ou externos (BOTHOS, APOSTOLOU, MENTZAS, 2009) e os tomadores de decisão (BOTHOS; APOSTOLOU; MENTZAS, 2009; PEREZ; LARRINAGA; CURRY, 2015).

Ao final desta fase analítica e decisiva tem-se um portfólio com ideias selecionadas e priorizadas (número de ideias é reduzido) e as avaliações são recebidas.

\subsection{Refinamento de ideias}

Alessi et al (2015) descreve que o resultado desta fase é a transformação da ideia em um produto/serviço sustentável em termos tecnológicos, econômicos e sociais. Para isso podese seguir as considerações feitas nas fases anteriores e buscar novas informações.

Na mesma linha de pensamento Aagaard (2013) traz o termo "Maturidade da Ideia" e descreve que "curiosamente", o conceito de maturação de ideias não é abordado na literatura da mesma forma que as demais etapas. Na prática, a maturação das ideias geralmente inclui testes, prototipagem e construção de um business case como forma de melhorar e desenvolver.

A mesma autora descreve ainda que o resultado da maturação da ideia, dentro do processo inicial da inovação é um conceito bem definido de produto, ou seja, ter clareza e estar alinhado com as necessidades do cliente. Ela argumenta ainda que é necessário separar ideias de "conceitos de produtos", pois ideias podem permanecer livres para serem remodeladas, já um conceito é o início de um processo de desenvolvimento (AGGARD, 2013).

Desta forma, neste artigo entende-se que a ideia maturada e refinada não é ainda a formulação de um conceito de produto, pois ela ainda pode sofrer alterações e não entrou na linha de produção. Além disso, um conceito de produto, "exige uma definição de produto (explícito e estável) e um plano de projeto (prioridades, planos de recursos e cronogramas de projetos)", como afirmou Khurana e Rosenthal (1997, p.106).

Os principais atores envolvidos são os especialistas de domínio, pois são os que detêm conhecimento específico sobre a ideia. Como resultado tem-se um portfólio das ideias detalhadas daquelas que foram selecionadas e priorizadas. As ideias neste momento estão prontas para serem transformadas em conceitos, e assim, avançar para o desenvolvimento de produto.

Perspectivas em Gestão \& Conhecimento, João Pessoa, v. 10, n. 3, p. 205-225, set./dez. 2020. 


\subsection{Armazenamento de ideias}

O armazenamento das ideias acontece durante todas as fases. Em 1983 Green, Bean e Snavely em seu modelo de gestão de ideias, já previam a necessidade de algum repositório para ideias que além de reter pudesse facilitar a recuperação destas ideias. $O$ armazenamento deve prover os meios para arquivar, procurar e permitir a rastreabilidade das ideias pelos autores certos no momento certo.

O armazenamento facilita a criação de um portfólio de ideias. Gallmeister e Lutz (2016) consideram o armazenamento de ideias no sentido da criação de um portfólio final de ideias para a distribuição entre as equipes que tem a capacidade de desenvolvimento. Portanto, entende-se que as ideias selecionadas durante o processo devem estar armazenadas para serem distribuídas as pessoas-chave na organização para continuar o processo de maturação e seu possível desenvolvimento ou implementação.

Porém, não só as ideias selecionadas para este portfólio devem ser armazenadas, mas também fragmentos de ideias não selecionados devem ser armazenados para uso futuro, uma vez que estas ideias podem ser recicladas e remodeladas e até "vendidas" para demais organizações (BREM, VOIGT, 2006, GALLMEISTER, LUTZ, 2016).

Visando o armazenamento, Duin et al (2010) criaram um modelo de entidade relacionamento, no qual especificam itens que devem estar registrados e armazenados juntos com as ideias. Alguns deles são:

- Marcação - são armazenadas qualquer tipo de tag associadas a uma ideia fornecida pelo usuário;

- Anexo - qualquer tipo de anexo associado a uma ideia deve ser armazenado;

- Pontuação- grupo de ideias que estão sujeitos à classificação devem ter o seu resultado armazenado junto com as ideias;

- Avaliação - grupo de ideias avaliadas devem ter seus resultados armazenados com as ideias;

- Comentários - qualquer comentário fornecido por um usuário do repositório de ideias deve ser armazenado com a respectiva ideia;

- Fórum - ideias podem estar sujeitas a discussão, portanto, as postagens de fóruns, mesmo que na web, devem ser armazenados juntamente com seus interessados;

- Nome de organizações e usuários - devem ser armazenados com as respectivas ideias relacionadas

- Competências - O perfil de competência deve ser armazenado junto a cada usuário.

Para Sandström e Björk (2010) deve-se criar um banco de ideias, no qual, por meio de um SGI este seja capaz de aprender com as ideias antigas já desenvolvidas e utilizá-las em projetos vindouros, bem como inspirar pessoas no seu trabalho diário. Por exemplo, quando um projeto é iniciado, é indicado nas especificações que o banco de dados de ideias deva ser pesquisado por ideias relevantes daquela área.

Apesar de Muhah et al (2013) não destacarem a fase de armazenamento, eles citam a reutilização de ideias armazenadas para que haja o surgimento de outras. $O$ armazenamento

Perspectivas em Gestão \& Conhecimento, João Pessoa, v. 10, n. 3, p. 205-225, set./dez. 2020. 
também deve facilitar a fase de enriquecimento, pois estas devem estar acessíveis para que aconteça a atividade colaborativa.

Como resultado desta fase tem-se as ideias armazenadas juntamente com seus descritores. Os dados armazenados com as ideias bem como as próprias ideias evoluem ao longo do processo. O banco de ideias é acionado durante vários momentos no decorrer do processo como na geração, enriquecimento, avaliação e seleção.

\subsection{Feedback e Acompanhamento}

O feedback permite que o processo seja retroalimentado, fazendo com que haja um fluxo contínuo de ideias sendo criadas e melhoradas. Para Gallmeister e Lutz (2016) é fundamental que todos os participantes que geraram e melhoram as ideias recebam feedback. É importante que o feedback seja oportuno, relevante e honesto. Caso contrário, as pessoas logo irão parar de dar ideias (GALLMEISTER; LUTZ, 2016).

Neste sentindo, o comportamento dos participantes deve ser recompensado. A recompensa não precisa ser grande, o mais importante é que todas as ideias sejam recompensadas para manter a motivação dos participantes (GERLACH, BREM, 2017). As recompensas podem ser tanto intrínsecas quanto extrínsecas e quando um grupo participa é necessário já estar prevista na política de funcionamento se eles serão recompensados iguais ou não (GERLACH, BREM, 2017).

O monitoramento, permite avaliar e monitorar o sucesso ou a falha do programa como um todo, por meio também de feedback, só que agora recebido dos usuários com relação ao programa de gestão de ideias (ALESSI et al., 2015). Os autores ainda colocam que as técnicas que eles utilizam são: monitoramento por questionários, entrevistas, pesquisas e revisões coletados pelo SGI.

No monitoramento é realizado também pela construção de métricas do programa que além de monitorar e avaliar a eficácia do programa, mantém a gestão da organização informada e comprova o sucesso do programa e identifica os pontos fracos. Elas podem estar divididas em:

1) Métricas especificas do processo da ideia: Taxa de participação; Número de ideias sugeridas; números de ideias selecionadas; taxa de implementação; tempo geral de processamento; extensão as recompensas.

2) Métricas da ideia: diversidade; número de ideias externas; número de ideias com alta qualidade; número de ideias com qualidade média; variação da qualidade da ideia; número de ideias de qualidade entre as unidades da organização; número de ideias de qualidade de fontes externas; número de ideias que não teriam sido realizadas sem a Gl.

3) Métricas do resultado de um programa: redução de custos; relação total de benefícios; receita das ideias; número de ideias que viraram produtos; número de meses para comercialização; qualidade do produto.

Esta fase é de responsabilidade do gestor do programa (GALLMEISTER, LUTZ, 2016). No entanto, participam da troca toda comunidade de usuários, uma vez que há os que dão e os que recebem o feedback (ALESSI et al. 2015).

\subsection{Discussão sobre as fases e atores identificados}

As fases identificadas: Preparação; Geração e Coleta; Filtragem e Agrupamento; Enriquecimento; Avaliação e seleção, bem como Armazenamento e Feedback, podem ser

Perspectivas em Gestão \& Conhecimento, João Pessoa, v. 10, n. 3, p. 205-225, set./dez. 2020. 
analisadas como um processo contínuo e cíclico, onde cada etapa depende da etapa anterior ou que caminham paralelamente como nos casos do armazenamento e feedback.

É possível observar que este processo favorece o acompanhamento da evolução das ideias desde a geração até o refinamento. Após a ideia passa para a etapa de conceitualização que, como definido por Koen et al (2001) é a última etapa do front end da inovação. Todas essas atividades devem ser geridas por meio de um processo a fim de evitar erros que possam tornar ineficiente o processo de inovação e ao final ocasionar produtos menos assertivos.

Além disso, observa-se que os atores envolvidos variam de cliente, colaboradores internos, a comunidade, fornecedores e demais interessados. Estes atores podem assumir diferentes papéis dependendo da fase, como resume o Quadro 4. No Quadro 4 também é possível observar que a função de especialistas e do gestor atua em mais de uma fase com certa frequência. No entanto, apesar da existência deles uma definição mais clara sobre as competências exigidas ainda não está suficientemente apoiada na literatura.

Quadro 4: Atores e funções do processo de gestão de ideias

\begin{tabular}{|c|c|c|c|c|c|c|}
\hline & Ideador & Especialista & $\begin{array}{l}\text { Tomador } \\
\text { de } \\
\text { decisão }\end{array}$ & $\begin{array}{l}\text { Gestor/ } \\
\text { comitê }\end{array}$ & Colaborador & Avaliador \\
\hline \multicolumn{7}{|l|}{ Preparação } \\
\hline \multicolumn{7}{|l|}{ Geração e coleta } \\
\hline \multicolumn{7}{|l|}{$\begin{array}{l}\text { Filtragem, } \\
\text { agrupamento } \\
\text { categorização }\end{array}$} \\
\hline \multicolumn{7}{|l|}{ Enriquecimento } \\
\hline \multicolumn{7}{|l|}{$\begin{array}{l}\text { Avaliação, seleção } \\
\text { e priorização }\end{array}$} \\
\hline \multicolumn{7}{|l|}{ Refinamento } \\
\hline \multicolumn{7}{|l|}{$\begin{array}{l}\text { Feedback e } \\
\text { acompanhamento }\end{array}$} \\
\hline Armazenamento & & & & & & \\
\hline
\end{tabular}

Fonte: Elaborado pelos autores com base na literatura estudada

Por fim observa-se que o processo de gestão de ideias possui fases que são divergentes e convergentes as quais alternam-se durante o processo. Nas fases divergentes, como a geração e o enriquecimento, técnicas criativas e colaborativas devem ser fortemente estimuladas. Já nas fases convergentes, como a filtragem, agrupamento, avaliação e seleção, a visão analítica e racional deve ser priorizada provendo equilíbrio entre o formal e informal.

\section{CONSIDERAÇÕES FINAIS}

O presente artigo teve como objetivo identificar na literatura os elementos comuns que caracterizam um processo de gestão de ideias com foco no Front end da inovação. Podese notar que há consenso entre autores de que a gestão de ideias deve ser feita por meio de fases e atividades que se inter-relacionam. O objetivo do trabalho foi alcançado, uma vez que se apresentou os elementos divididos em fases e funções/atores que permitiram caracterizar o

Perspectivas em Gestão \& Conhecimento, João Pessoa, v. 10, n. 3, p. 205-225, set./dez. 2020. 
processo de gestão de ideias. As fases foram: preparação, geração e coleta, filtragem e agrupamento, enriquecimento, avaliação e seleção, refinamento, armazenamento e, por fim o feedback e monitoramento. Referente aos atores eles podem ser: funcionários e colaboradores internos, fornecedores, usuários e consumidores, bem como especialistas da área acadêmica, a comunidade em geral e demais interessados. Estes atores podem assumir diferentes funções no decorrer do processo, como: gestor da ideia, especialista, ideador, colaborador, avaliador e tomadores de decisão.

$\mathrm{O}$ artigo contribui para a teoria fazendo um aparato geral dos principais modelos e a identificação de elementos comuns entre eles. Como a literatura já vinha apontando os modelos que estruturam a gestão de ideias são, em parte, baseados em sistemas de gestão de ideias. Dos 16 modelos analisados 10 deles têm como foco a proposição de estruturação para sistemas de gestão de ideias. Estes sistemas têm como tendências a utilização de tecnologia semântica para o tratamento das ideias e colaborativa para o enriquecimento delas.

Pode-se observar fases consolidadas referem-se a geração, enriquecimento, e avaliação e seleção. Desta maneira, entende-se como oportunidade de aperfeiçoamento as demais fases e como elas se relacionam com as mais consolidadas para assim ter-se um processo mais coeso. Como exemplo na fase de preparação, pode-se investigar: o que a organização precisa realizar para iniciar um programa de gestão de ideias, quais pessoas e departamentos e recursos serão envolvidos, quais fontes de conhecimento precisam ser consultados e mapeados.

Também o pré-tratamento destas ideias, como exemplo a filtragem, agrupamento e categorização por meio de tecnologias semânticas. Ainda que indispensável e presentes em todos os modelos que trabalham com sistemas de gestão de ideias o armazenamento dentro destes modelos pode ser melhor explorado para ir além de um repositório de dados e ideias e sim de conhecimento, ou seja, como tornar o banco de ideias um repositório de conhecimento acessível a todos da organização. Neste sentindo, o papel da gestão do conhecimento como apoiadora a gestão de ideias também pode ser explorado em pesquisas futuras

Ainda com relação as fases, o feedback e como ocorre o acompanhamento dessas iniciativas carece de estudos. Nesse sentindo, como avaliar o sucesso das iniciativas, quais métricas utilizar e qual modelo utilizar são possibilidades de pesquisas futuras.

$\mathrm{O}$ artigo também trouxe a importância dos atores envolvidos durante o processo. $\mathrm{Na}$ qual observa-se oportunidades de trabalho futuros como por exemplo explorar a formação de rede de atores tanto para gerar, enriquecer e selecionar ideias para que elas possam serem levadas adiante, bem como definir as competências necessárias para cada função.

Por fim, esses assuntos são campos para estudos futuros, bem como pesquisas de campo que agreguem informação a teoria estudada. As limitações deste trabalham ficam na estratégia de busca utilizada e critérios utilizados para análise.

\section{AGRADEDIMENTOS}

Agradecimento ao órgão fomentador desta pesquisa: Coordenação de Aperfeiçoamento de Pessoal de Nível Superior (CAPES).

\section{REFERÊNCIAS}

AAGAARD, Annabeth. A theoretical model of supporting open source front end innovation through idea management. International Journal of Business Innovation and Research, v. 7, n. 4, p. 446-465, 2013. 
ALESSI, Marco et al. Applying Idea Management System (IMS) approach to design and implement a collaborative environment in public service related open Innovation processes. Complex Systems Informatics and Modeling Quarterly, n. 5, p. 26-38, 2015.

AMABILE, Teresa M. How to kill creativity. Boston, MA: Harvard Business School Publishing, 1998.

BESSANT, J.; LAMMING, R.; NOKE, H.; PHILLIPS, W.; Managing innovation beyond the steady state. Technovation, Volume 25, N. 12, 2005, p. 13661376.

BJÖRK, J., BOCCARDELLI, P.; MAGNUSSON, M. G. Ideation capabilities for continuous innovation. Creativity e Innovation Management, Volume 19, Number 4, 2010.

BOEDDRICH, H. J. Ideas in the workplace: a new approach towards organizing the fuzzy front end of the innovation process. Creativity and innovation management, 2004, 13(4), 274-285.

BOTHOS, Efthimios; APOSTOLOU, Dimitris; MENTZAS, Gregoris. Collective intelligence for idea management with Internet-based information aggregation markets. Internet Research, v. 19, n. 1, p. 26-41, 2009.

BRENNAN, A., \& DOOLEY, L. Networked creativity: a structured management framework for stimulating innovation', Technovation, Vol. 25, No. 12, pp.1388-1399, 2005.

BREM, Alexander; VOIGT, Kai-Ingo. Integration of market pull and technology push in the corporate front end and innovation management-Insights from the German software industry. Technovation, v. 29, n. 5, p. 351-367, 2009

CHESBROUGH, Henry W. The era of open innovation. Managing innovation and change, $v$. 127, n. 3, p. 34-41, 2006.

COOPER, Robert G. The new product process: a decision guide for management. Journal of Marketing Management, v. 3, n. 3, p. 238-255, 1988.

DE SOUZA, Marcela Tavares; DA SILVA, Michelly Dias; DE CARVALHO, Rachel. Revisão integrativa: o que é e como fazer. Einstein, v. 8, n. 1 Pt 1, p. 102-6, 2010.

EL BASSITI, Lamyaa; AJHOUN, Rachida. Semantic-Based Framework for Innovation Management. In: European Conference on Knowledge Management. Academic Conferences International Limited, 2014. p. 1173.

FLYNN, M., DOOLEY, L., O'SULLIVAN, D., \& Cormican, K. Idea management for organisational innovation. International Journal of innovation management, 7(04), 417-442, 2003.

GALLMEISTER, Ute; LUTZ, Birgit. Engagement and Retention: Essentials of Idea Management. Handbook of Human Resources Management, p. 727-745, 2016.

GALBRAITH, Jay R. Designing the innovating organization. Organizational dynamics, v. 10, n. 3, p. 5-25, 1982.

GAMBATESE, J. A.; HALLOWELL, M.; Enabling and measuring innovation in the construction industry. Construction Management and Economics, Volume 29, Issue 6, 2011, Pages 553-567.

Perspectivas em Gestão \& Conhecimento, João Pessoa, v. 10, n. 3, p. 205-225, set./dez. 2020. 
GERLACH, Sophia; BREM, Alexander. Idea management revisited: A review of the literature and guide for implementation. International Journal of Innovation Studies, v. 1, n. 2, p. 144-161, 2017.

GREEN, Stephen G.; BEAN, Alden S.; SNAVELY, B. Kay. Idea management in R\&D as a human information processing analog. Human Systems Management, v. 4, n. 2, p. 98-112, 1983.

HORTON, G., GOERS, J. Mining Hidden Profiles in the Collaborative Evaluation of Raw Ideas. System Sciences (HICSS), 2014.

IVERSEN, Håkon et al. Idea management: A life-cycle perspective on innovation. In: 2009 IEEE International Technology Management Conference (ICE). IEEE, 2009. p. 1-8.

JENSEN, Anna Vagn. A literature review of idea management. In: DS 71: Proceedings of NordDesign 2012, the 9th NordDesign conference, Aarlborg University, Denmark. 22-24.08. 2012. 2012.

KOEN, P. A. et al. Providing clarity and a common language to the "fuzzy front end". Research Technology Management, Arlington, v. 44, n. 2, p. 46-55, 2001.

KOEN, P. A., BERTELS, H. M.J., KLEINSCHMIDT, E. J. Managing the Front End of Innovation-Part II: Results from a Three-Year Study: Effective Front-End activities were found to be significantly different for incremental and radical projects. Research-Technology Management. V. 57, n.3, p. 25-35, 2014

MESSERLE, M.; BINZ, H.; ROTH; D.; Elaboration and assessment of a set of criteria for the evaluation of product ideas. 19th International Conference on Engineering Design. Volume 9, 2013.

MURAH, Mohd Zamri et al. Kacang Cerdik: A Conceptual Design of an Idea Management System. International Education Studies, v. 6, n. 6, p. 178-184, 2013.

PAHL, G., BEITZ, W., FELDHUSEN, J., Grote, K.-H. Engineering Design: A systematic approach, 2a ed. Springer, 2007. $629 \mathrm{p}$.

PEREZ, Alain; LARRINAGA, Felix; CURRY, Edward. The Role of Linked Data and SemanticTechnologies for Sustainability Idea Management. In: International Conference on Software Engineering and Formal Methods. Springer, Cham, 2013. p. 306-312.

SANDSTROM, Christian et al. Idea management systems for a changing innovation landscape. International Journal of Product Development, v. 11, n. 3-4, p. 310-324, 2010.

SINT, Rolf et al. Ideator-a collaborative enterprise idea management tool powered by KiWi. In: Fifth Workshop on Semantic Wikis Linking Data and People 7th Extended Semantic Web Conference Hersonissos, Crete, Greece. 2010. p. 41.

STEVANOVIC, M.; MARJANOVIC, D.; STORGA, M. Idea relevancy assessment in preparation of NPD. In: Proc. of the 19th International Conf. of Engineering Design, Sungkyunkwan University, Seoul, Korea. 2013.

SUKHOV, A.; The role of perceived comprehension in idea evaluation. Creativity e Innovation

Perspectivas em Gestão \& Conhecimento, João Pessoa, v. 10, n. 3, p. 205-225, set./dez. 2020. 
Management. Volume1, N.13, 2018.

TORRACO, Richard J. Writing integrative literature reviews: Guidelines and examples. Human resource development review, v. 4, n. 3, p. 356-367, 2005.

VOIGT, Kai-ingo; BREM, Alexander. Integrated idea management in emerging technology ventures. In: 2006 IEEE International Conference on Management of Innovation and Technology. IEEE, 2006. p. 211-215.

WESTERSKI, Adam; IGLESIAS, Carlos A.; NAGLE, Tadhg. The road from community ideas to organisational innovation: a life cycle survey of idea management systems. 2011.

XIE, Luning; ZHANG, Pengzhu. A three phases idea selection approach for team creation. In: 2008 International Seminar on Business and Information Management. IEEE, 2008. p. 326329.

REINERTSEN, Donald G.; SMITH, Preston G. The strategist's role in shortening product development. Journal of Business Strategy, v. 12, n. 4, p. 18-22, 1991.

Artigo recebido em 18/07/2019 e aceito para publicação em 07/12/2020

Perspectivas em Gestão \& Conhecimento, João Pessoa, v. 10, n. 3, p. 205-225, set./dez. 2020. 